\title{
Low-Power IoT Devices for Measuring Environmental Values
}

\author{
George Suciu, Ana Lavinia Petrache, Cristina Badea, \\ Ijaz Hussain \\ R\&D Department \\ Beia Consult International \\ Bucharest, Romania \\ george@beia.ro
}

\begin{abstract}
This paper presents how Pycom, Waspmote and Raspberry $\mathrm{Pi}$ devices meet low power requirements as part of Internet of Things (IoT). These technologies improve scalability, efficiency and optimize the structure responding to known IoT drawbacks such as low energy, resources and slow information processing. They are part of applications included in Smart City, Smart Home, Smart Grid, mainly telemetry. Libelium technology, including the Waspmote devices, are presented in this paper. Pycom has a variate set of devices: Pysense having five integrated sensors: temperature, humidity, altitude, pressure and light, LoPy, LoPy4 microcontrollers, Pytrack, SiPy, FiPy, GPy, WiPy, expansion boards. Raspberry Pi is known for being a single board-computer which is running its own operating system (OS). It consumes more power, unless running processes are prioritized for lowering the consumption, which requires a vast Linux knowledge. In this article we present an experiment using Pycom and Pysense which will show its capability for collecting data from the environment. Also, this paper describes how the montage works with Adafruit cloud. Pycom devices functioning in low-power wide-area networks use long range (LoRa) antennas which are especially designed for such networks. This type of antenna helps Pycom montages to save energy and for this to take effect, it must be placed in a LPWAN network. LoRa runs well in exposed environments, but connection can be affected by buildings. Having 14dBm output power, LoRa covers $25 \mathrm{~km}$ flat areas.
\end{abstract}

Keywords-Pycom, Pysense, LoRa, Waspmote, Libelium

\section{INTRODUCTION}

The environmental changes affect entire ecosystems and data is needed in order to analyze and model these changes. There is a growing number of sensing devices connected to the Internet, called IoT, which can provide this kind of data.

As an example, Pycom is known for its optimized lowpower structure, especially Pysense with its integrated sensors [1], as it is easier to install and data gathering is more reliable. IoT can be useful in small places such as rural areas where it can monitor humidity or water level to tell if an area is flooded or under draught. Also, it runs well in exposed environments, but connection can be affected by buildings.

This system under test consists of a Pysense sensor board connected to Pycom through I2C interface with the following integrated sensors: 3-axis accelerometer, light sensor, humidity and temperature sensor, barometric pressure sensor.

\author{
Tony Buteau, David Schlachet, Loic Durand, Matthieu \\ Landez \\ IMT Atlantique Brest \\ INP Bordeaux Aquitaine \\ France \\ proiecte@beia.ro
}

The system connects to the Internet network through Wi-Fi, GSM, Bluetooth or LoRa [2] and can use MQTT [3] protocol to send data to different cloud platforms.

Furthermore, data collected by sensors can be locally processed to issue warnings or sent to a destination for further use. In our work the Pycom devices use Adafruit cloud [4] to send data, which can be accessed using a web browser. A user will register to this site, create a feed for each sensor, a dashboard and then create a chart using each feed where we can see results from sensors. This system can run without connection to a PC, while powered to a battery or solar panel, but libraries must be uploaded first.

The paper is structured as follows: Section II presents methods and related work, Section III analyzes measurement results, while Section IV presents the conclusions.

\section{METHODS AND RELATED WORK}

In our measurement method for environmental values, the time was calculated to execute functions using central processing unit (CPU) cycles between two calls. Table 1 shows the time measured on two devices, Waspmote and Raspberry $\mathrm{Pi}$, the configuration of the module, joining a network and finally send the data through.

As shown in the table, Raspberry $\mathrm{Pi}$ has the fastest connections which cost less energy than a Waspmote WiFi connection.

TABLE I. TIME MEASUREMENT ON TWO DEVICES

\begin{tabular}{|c|c|c|}
\hline Phase & Waspmote $[\mathbf{m s}]$ & Raspberry Pi [ms] \\
\hline Turn On & $2500 \mathrm{~ms}$ & $<1000 \mathrm{~ms}$ \\
\hline WiFi Setup & $9196 \mathrm{~ms}$ & $<1000 \mathrm{~ms}$ \\
\hline Join Network & $2879 \mathrm{~ms}$ & $<1000 \mathrm{~ms}$ \\
\hline $\begin{array}{c}\text { Sending HTTP } \\
\text { message }\end{array}$ & $1353 \mathrm{~ms}$ & $<1000 \mathrm{~ms}$ \\
\hline $\begin{array}{c}\text { Sending TCP } \\
\text { message }\end{array}$ & $7421 \mathrm{~ms}$ & $<1000 \mathrm{~ms}$ \\
\hline $\begin{array}{c}\text { Sending UDP } \\
\text { message }\end{array}$ & $6286 \mathrm{~ms}$ & \\
\hline
\end{tabular}

Experiments made on Waspmote devices proved its low power capabilities. Waspmote sent data non-stop for 42.74 
hours, proving the capacity of its battery. In this way some issues were found with battery reading function which was not showing the real value.

Waspmote's sleep state determines an instantly decrease of current to $57.3 \mu \mathrm{A}$, however hibernate state isn't recommended because it takes 11 seconds to have this current value.

\section{A. Pycom/Pysense}

This montage was chosen for the ease of installation, its functionalities and the need to monitor the environment and visualize the data obtained. Reliable transmission of gathered data from sensors to the server is needed. Pysense has five integrated sensors: a third-axis accelerometer, a light sensor, a humidity/temperature sensor and a barometric pressure sensor. They are useful in small places. Displaying overflow warnings is possible by placing water sensors along a river [5].

In rural areas humidity level can be monitored for announcing flood. This can be predicted with the help of Pysense and Adafruit displaying on our computers modifications in the atmosphere (Fig. 1). Water level data can be received by placing more water motes vertically [6].

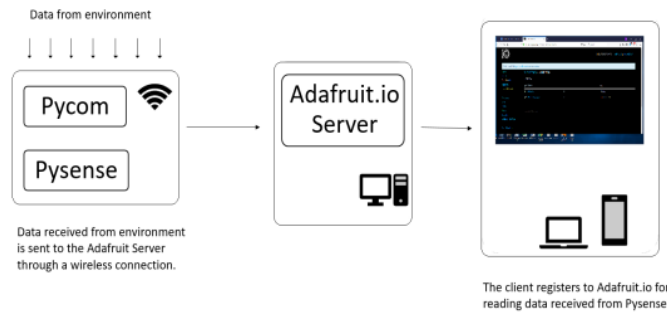

Fig. 1 Mode of operating

In the next example is presented a montage having Pysense connected with Pycom through an $\mathrm{I}^{2} \mathrm{C}$ (InterIntegrated Circuit) interface. Pycom/Pysense connects to network through WiFi due to the fact that our network infrastructure is suited to $\mathrm{WiFi}$.

The MQTT (Message Queuing Telemetry Transport) protocol can be used to send some data in Cloud. Also, Pycom's media access control (MAC) address must be known by the network's portal. Pycom devices use Adafruit cloud. This can be accessed on https://io.adafruit.com. Pycom's user will register to this site, will create a feed for each sensor and a dashboard and then will create a chart using each feed where we can see results from sensors. This montage can run without a computer, but libraries must be uploaded to the device first.

Pycom devices support maximum $5 \mathrm{~V}$ and can be powered with LiPo (lithium polymer) batteries, or through USB 5V charger.

\section{B. WiFi in IoT}

Low-Power WiFi is an alternative for the 802.11 specification with functions for saving power, ensuring data transmission between WiFi devices don't need any additional interfaces for network connections [7]. WiFi and LoRa work in star topologies [8].

WiFi uses known protocols, so low power devices can send data easier without being necessary to translate standards for connecting to Internet. TCP (Transmission Control Protocol) is used often by these devices. Having low power means a WiFi antenna of a low power device covers smaller areas [7].

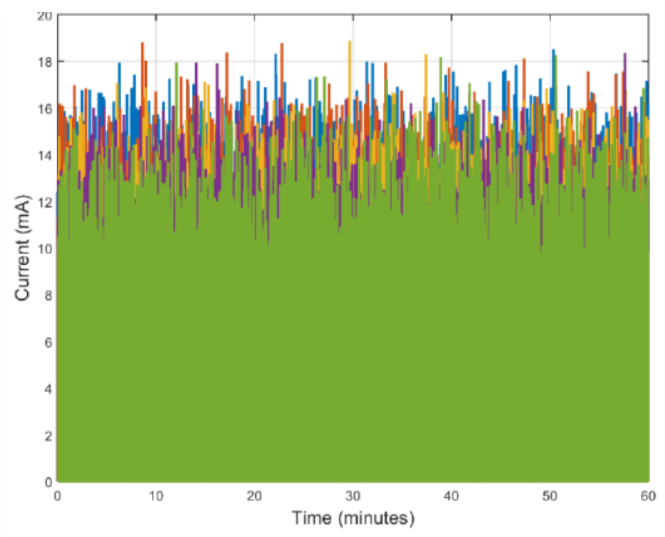

Fig. 2 WiFi Power consumption

On Raspberry Pi, Ethernet power consumption during download increases and it reaches maximum $70 \mathrm{~mW}$. When data rate is $50 \mathrm{Mbps}$, power drops instantly due to Raspberry Pi's hardware structure. WiFi power consumption for downloading is minimum $0.95 \mathrm{~W}$ and maximum $1.4 \mathrm{~W}$ for a high traffic. The variance on WiFi is low [8]. For saving battery power, the WiFi module needs to be as fast as possible on Libelium, Raspberry Pi or Pycom devices [7]. The graph from Fig. 2 shows how much power WiFi connection consumes in a given duration of 1 hour.

Bluetooth and WiFi present no considerable increases of current draw when the other devices in the same network or bandwidth or when the transmission rate is altered.

Fig. 3 illustrates an increase of peak consumption during WiFi transmission. There will be a higher power spike for an incoming transmission occurring at the same time with this connection.

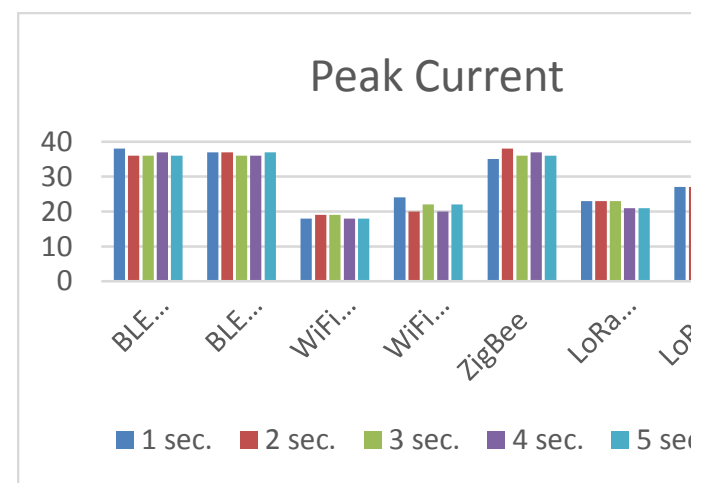

Fig.3 WiFi Peak Currents messaging periods 
For a good connection, WiFi device must be placed at 9 meters from the antenna or maximum 12.5 meters. If it is placed further, there will be packet loss. The router can connect through $\mathrm{WiFi}$ to 50 devices simultaneously.

Fig. 4 shows the package success rate depending on distance. The WiFi range is maximum 20 meters. Within 10 meters, packages aren `t lost.

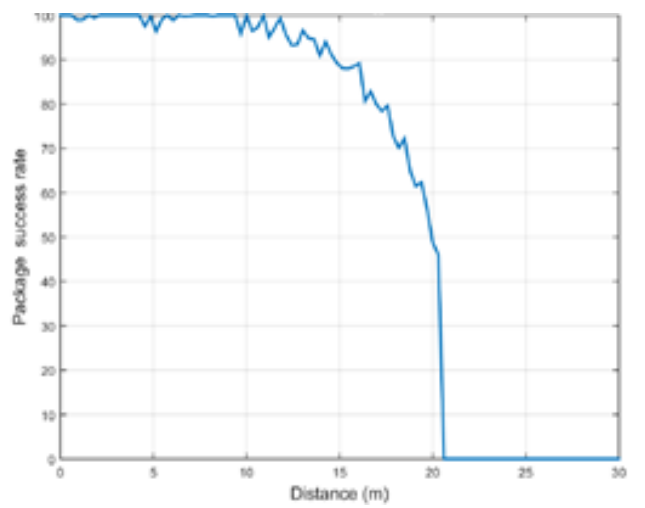

Fig. 4 WiFi Experimental Range and Package success Rate

\section{Estimote}

This experiment shows how Estimote BLE beacons function in various conditions. It takes place in a single $9 \mathrm{mx} 11 \mathrm{~m}$ room. There are some obstacles aside from tables and chairs. There is a single BLE beacon in this room at a given time to avoid interference. For measuring and calculating we used a Google Nexus 5 device running an Android application that uses an AltBeacon Android library. Accurate distance was obtained with a measuring tape.

Estimote is tested five times or iterations. Experimental results (Fig 5) are shown in the following graph: the RSSI value influenced by filters.

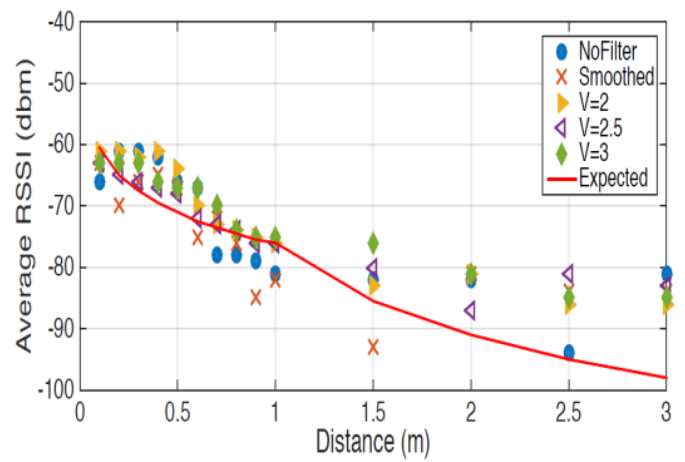

Fig. 5 Average RSSI value of the beacons

In first iteration is obtained the raw received signal strength indicator (RSSI) values and resulting distance calculations without smoothing or filtering. The second iteration: RSSI values are smoothed over a twenty second interval, eliminating the top and bottom $10 \%$ of values.

Third iteration measures the incoming RSSI signals and apply a simple Kalman filter of varying Kalman values (2, 2.5, and 3). Within each iteration, 14 measurements are taken. The beacon remains still on a table, while the smartphone moves along the table at the following set of distances: $0.1,0.2,0.3$, $0.4,0.5,0.6,0.7,0.8,0.9,1.0,1.5,2.0,2.5,3.0$ meters. At each distance, the RSSI and corresponding distance is recorded. All measurements are given 20 seconds to calibrate before recording.

\section{MEASUREMENTS RESULTS}

In this section we present the measurement results from the low-power IoT devices recorded using time series data bases.

\section{A. Grafana}

The data recorded by sensors using Raspberry pi are sent to Grafana [10], as presented in Fig. 6. The monitoring of transmission of data to Grafana is done using mqtt-spy [11].

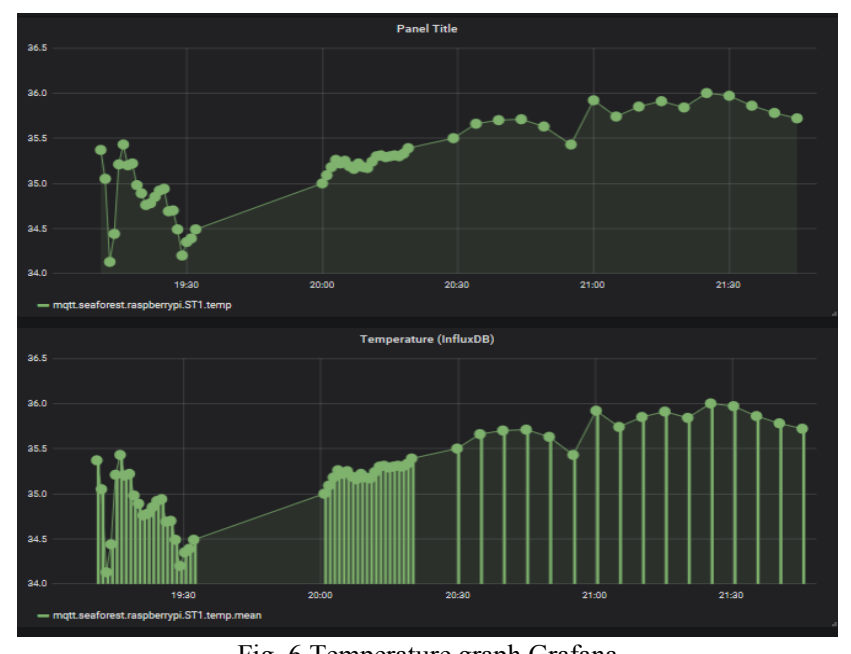

Fig. 6 Temperature graph Grafana

In the above image is presented two graphs representing the data collected from the temperature sensor. The data are recorded within 6 hours. The data are in Celsius degrees and are recorded at 5 minutes. Such platforms can be used for measuring energy efficiency of large scale systems [12].

\section{B. Graphite}

To view the data from the temperature and gas sensor we used Graphite [13], which is a suite of software used for storing, collecting, and presenting time data. For efficient performance, it is necessary to collect and transfer data through other applications that retransmit data in the format supported by Graphite.

Graphite is used by customers to track the performance of sites, applications, services, and servers. Graphite has been an innovation with regard to monitoring tools, making it easier to store, retrieve, share, and view data at certain time intervals. Many companies have implemented Graphite, which helps monitor the production of e-commerce services and the expansion plan.

In Fig. 7 we present temperature graphs while in Fig. 8 we present gas measurements during an hour, while the battery level showed an average decrease to $5 \%$. 


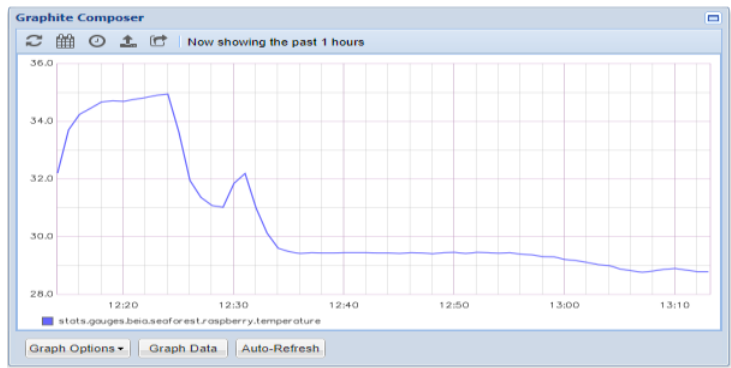

Fig. 7 Temperature graph

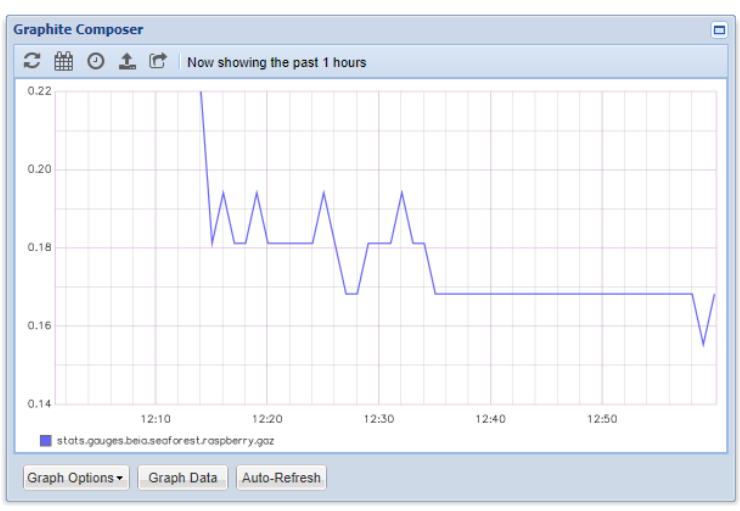

Fig. 8 Gas graph

Graphite has the following two functionalities:

1) Storing data in time-dependent series;

2) On request, they deliver the graphs of these data.

To send the data to Graphite, we need the StatsD library in the code.

StatsD can send any type of data to Graphite. StatsD receives Raspberry information and sends them to Graphite using UDP (User Datagram Protocol) protocol.

\section{Adafruit}

Pymakr package was installed using Atom compiler. In the project folder main.py, boot.py and mqtt.py along with the /lib folder are added. In /lib the user needs to place the libraries for sensors, pysense.py and pycoproc.py. The board connects to adafruit.io in order to send data.

To find the MAC address of the device, the user must write the following instructions in the Pymakr's console, when the board is connected:

- import network

- network.WLAN().mac()

- import ubinascii

- ubinascii.hexlify(network.WLAN().mac(),':').decode()

First, a dashboard is created where feeds will be placed. Adafruit IO uses 'feeds' to describe separate sets of data. For displaying graphs, the user creates a feed for each Pysense sensor.

In Fig. 12 we present the battery level of the device, measured and displayed using Grafana.

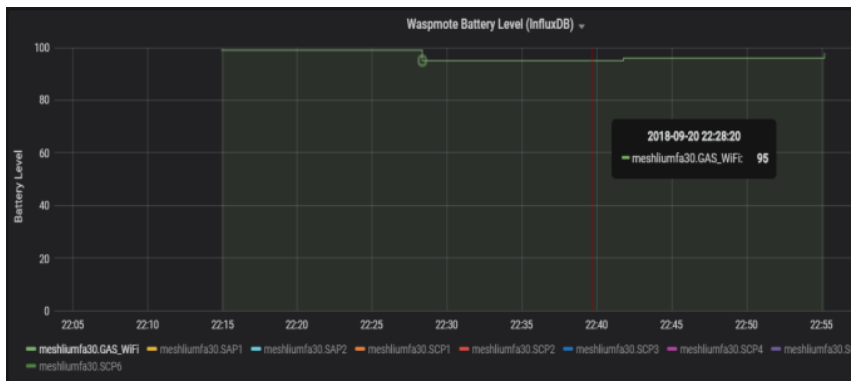

Fig. 9 Temperature graph

In Fig. 10, 11, 12 our experiments show three live charts that prove LoPy connection to Adafruit cloud was successful, while the battery level decreased again with an average of 5\% during one hour. As such, an environment can be monitored for long periods of time giving the chance to learn more about it.

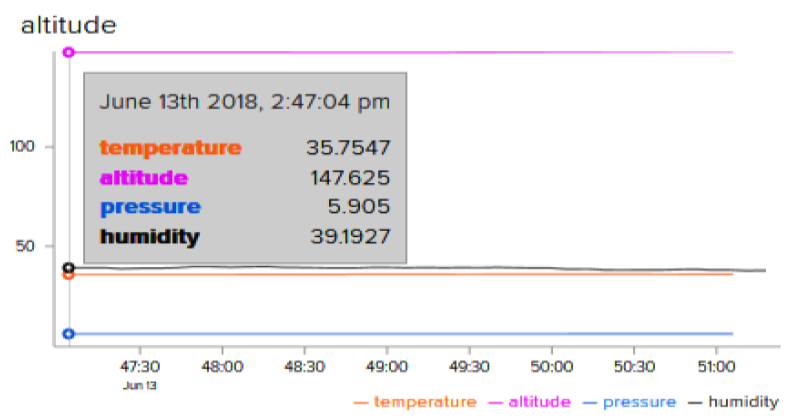

Fig. 9 Measuring the Altitude with Pysense

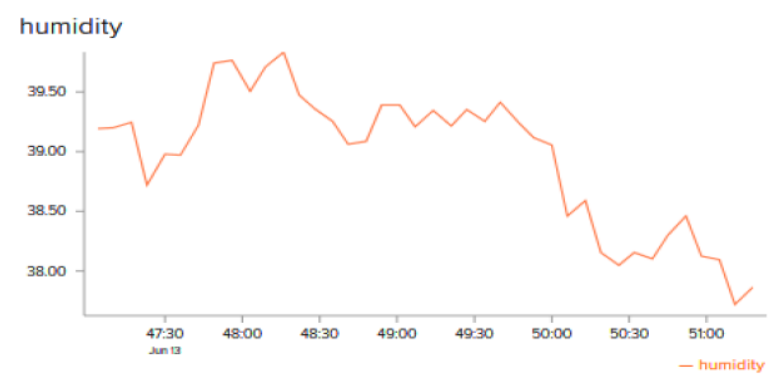

Fig. 10 Measuring the Humidity with Pysense

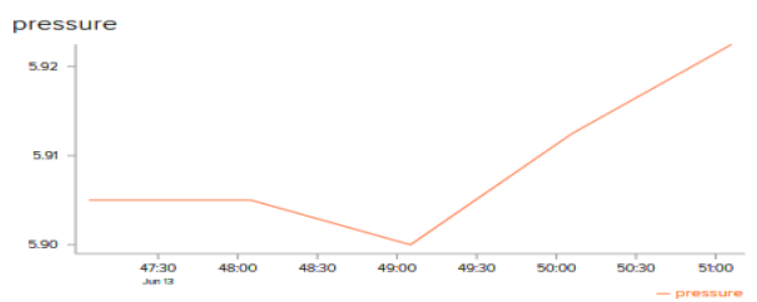

Fig. 11 Measuring the pressure with Pysense 1

The Adafruit graph displays data in real time and keeps data history. Those graphs are created by the user. There is a graph for every sensor, which can be easily created from Dashboard by clicking on Create New Block (the blue button right up corner) then select Line Chart. Here, the user can choose options from Show History menu. 


\section{CONCLUSIONS}

This paper has presented how devices such as Pycom, Waspmote and Raspberry $\mathrm{Pi}$ devices meet low power requirements as part of Internet of Things. We have presented that these technologies improve scalability, efficiency and will optimize the structure responding to known IoT drawbacks such as low energy, resources and slow information processing. Also, in this article, were described several methods of displaying the results as well as the energy consumption of some.

As future work we envision to deploy IoT devices powered by a solar panel and battery for measuring environmental values in a forest.

\section{ACKNOWLEDGMENT}

This work has been supported in part by UEFISCDI Romania and MCI through projects SeaForest, WINS@HI, TelMonAer, and funded in part by European Union's Horizon 2020 research and innovation program under grant agreement No. 777996 (SealedGRID project) and No. 787002 (SAFECARE project).

\section{REFERENCES}

[1] L. Hendrik, M. Rademacher, O. G. Aliu, K. Jonas. "Path Loss Models for Low-Power Wide-Area Networks: Experimental Results using LoRa", 2018.

[2] M. Cattani, C.A Boano, K. Römer. "An Experimental Evaluation of the Reliability of LoRa Long-Range Low-Power Wireless Communication." Journal of Sensor and Actuator Networks, Vol. 6, No. 2, 2017;

[3] R. K. Kodali, K. S. Mahesh. "A low cost implementation of MQTT using ESP8266." In Contemporary Computing and Informatics (IC3I), 2nd International Conference on, pp. 404-408. IEEE, 2016;

[4] D. K. Fisher, R. S. Fletcher, S. S. Anapalli, H. C. Pringle III. "Development of an Open-Source Cloud-Connected SensorMonitoring Platform." Advances in Internet of Things, Vol. 8, No. 1, 2017.

[5] H. Linka, M. Rademacher, O.G. Aliu and K. Jonas, "Path Loss Models for Low-Power Wide-Area Networks: Experimental Results using LoRa," 2018.

[6] A. Mackey and P. Spachos, "Performance Evaluation of Beacons for Indoor Localization in Smart Buildings,"School of Engineering, University of Guelph, Ontario, Canada.IEEE 2017.

[7] J.M.B. Ordinas and J.G. Vidal, "Low Power WiFi: Astudy on power consumption for Internet of Things, 2015.

[8] F. Kaup, P. Gottschling and D. Hausheer, "PowerPi: Measureing and Modeling the Power Consumption of the Raspberry Pi," $39^{\text {th }}$ Annual IEEE Conference on Local Computer Networks, 2014.

[9] M.C. Diego Rondon, J. David, W. Hernan, "Hardware node for sensing temperature and humidity in a Multiprotocol Wireless Sensor Network (WSN)"

[10] https://grafana.com/, 14.08.2018

[11] https://www.eclipse.org/paho/components/mqtt-spy/, 14.08.2018

[12] G. Suciu, L. Necula, V. Suciu, and Y. Curtmola. "Cloud-Based platform for enhancing energy consumption awareness and substantiating the adoption of energy efficiency measures within SMEs." In 14th IEEE International Wireless Communications \& Mobile Computing Conference (IWCMC), pp. 1018-1023, 2018.

[13] https://graphiteapp.org/, 14.08.2018 